\title{
Formulation of insoluble drugs. Study of pharmaceutical suspensions by response surface methodology
}

\author{
F. Nielloud ${ }^{1, *}$, G. Marti-Mestres ${ }^{1}$, R. Fortuné ${ }^{1}$, J.P. Mestres ${ }^{2}$ and H. Maillols ${ }^{1}$ \\ ${ }^{1}$ Laboratoire de Technique Pharmaceutique Industrielle, Faculté de Pharmacie, Avenue C. Flahault, \\ 34060 Montpellier Cedex, France \\ ${ }^{2}$ Laboratoire de Chimie Analytique, Faculté de Pharmacie, Avenue C. Flahault, \\ 34060 Montpellier Cedex, France
}

\begin{abstract}
The preformulation of insoluble drugs, sulfadiazine and aluminium hydroxyde, were studied in order to achieve a suspension with desirable requirements. The objective of the formulator is to avoid the irreversible aggregation called "caking", and to obtain a suspension with an airy, large volume sediment easily redispersible and with suitable rheological properties. An experimental design useful to determine optimal properties is a Central Composite Design. The surfactant, thickener and electrolyte at different proportions are the 3 factors studied. This strategy allows to point on the main significant effect and to determine the concentrations of each product leading to optimal properties of the suspensions.
\end{abstract}

Key words. Preformulation - suspension - experimental design - central composite design.

\section{Introduction}

Suspensions are dispersions of solids in liquids. Pharmaceutical suspensions are coarse dispersions of solid particles of different sizes $(0.1-10 \mu \mathrm{m})$ in a liquid medium, generally an aqueous solution. They are energetically unstable systems and are showing in some situations problems as aggregation of the single particles leading to "caking" (after the settling of particles to a closely packed sediment impossible to redisperse). Pharmaceutical suspensions have to be

* Correspondence and reprints.

Received January 27, 1998; revised July 3, 1998; accepted July 10, 1998. 
homogeneous and stable when the therapeutic doses are taken, so their formulation requires to avoid irreversible aggregation (cake) and to maintain the sediment in a flocculated state easy to disperse before dispensation. For this purpose, different agents can be used: surfactants for the wetting of the solids which improve their dispersion, thickeners for rheological requirements or electrolytes to modify the conditions of the electrolytic medium and increase the stability of the particles against aggregation $[1,2]$. The final aspect of the preparation, where rapid clearance of the supernatant is undesirable, is closely linked to the relative proportions of the constituents.

The aim of this work was to study the formulation of two insoluble drugs, an antimicrobial agent (the sulfadiazine) and an antacid product (the aluminium hydroxide), in order to achieve oral suspensions with optimal properties. It is unrealisable to examine the effects on the suspension properties of all components at various levels. Experimental designs offer an excellent approach to reduce the charge of time and money by limiting the number of experiments, furthermore this methodology gives a high quality of information. To determine the optimal experimental conditions, the response surface method can be employed. The approach we used is that of carrying out a Central Composite Design (CCD) in 3 factors. Several constituents were fixed, and the percentages of electrolyte, surfactant and thickener are the 3 main factors, of which the influence on the behaviour of suspensions is examined. These products $(\mathrm{NaCl}$ as electrolyte, Polysorbate 80 as surfactant and methylcellulose as thickener) have been chosen according to the litterature [3] and to medicines on the marketplace [4].

\section{Experimental}

\section{Materials and Methods}

The drugs are sulfadiazine, an antimicrobial agent (Merck, Darmstad, Germany), and aluminium hydroxide an antacid agent (Prolabo, Paris, France). The non-ionic surfactant used is a polysorbate 80 (Tween 80, ICI, Paris, France), the thickener a methylcellulose (Metolose, Shin-Etsu Chemicals, Seppic, Paris France) and the ionic species is $\mathrm{NaCl}$ (Merck). The vehicle is predetermined and constituted with $15 \%$ of saccharose (Prolabo), $0.5 \%$ of citric acid in water.

The preparation of $200 \mathrm{~g}$ of suspensions involved the simple mixing of the components at a temperature of $75{ }^{\circ} \mathrm{C}$. Suspensions were stocked in measuring glasses (vol. $250 \mathrm{~mL}$, precisely graduated) at $25{ }^{\circ} \mathrm{C}$ and then observed after 24 hours and 48 hours in the dark with a light allowing to read by transparency the dispersed sediment volume and the caking height. The rheological properties of suspensions were studied with a Rheometer (Rheomat 30, Contraves).

\section{Experimental strategy}

A central composite design was chosen to evaluate the factors that significantly influence suspension properties and what levels of the factors are needed to produce optimal suspension. This multivariate approach is composed of a full $2^{k}$ factorial ( $k$ is the number of variables) and of a star design. The first one is used to evaluate the effects of the variables and of their interactions. Then, six replicates at the centrepoint of the design and a pair of experiments along each co-ordinate axis allow to calculate the experimental error of the process and to determine response surfaces and the corresponding contourplots (Fig. 1). Accordingly, this CCD in three variables requires 20 experiments (Tab. II) [5-7]. The three factors are $X_{1}$, percentage of electrolyte, $X_{2}$, percentage of surfactant and $X_{3}$, percentage of thickener. A quadratic model corresponding to the following second order equation was built to describe the response:

$$
y=b_{0}+\Sigma_{i} b_{i} x_{i}+\Sigma_{i} b_{i i} x_{i}^{2}+\sum_{i j} b_{i j} x_{i} x_{j}
$$

where $y$ is the response, $x$ the factors and $b$ the coefficients of each term calculated by multiple regression analysis. The calculations are performed by the Statgraphics program [8].

The responses studied for the sulfadiazine were the volume of the dispersed sediment, $Y_{1}$, (in fraction of total volume), measured after 48 hours and the caking level, $Y_{2}$, (in millilitres) after 24 hours. The viscosity cannot be measured owing to the presence of the caking. For the aluminium hydroxide, the responses were the volume of the dispersed sediment, $Y^{\prime}{ }_{1}$, (in fraction of total volume), measured after 48 hours and as no caking appears after 24 hours we used as dependant variable the Oswald's parameter, $K$, obtained from Oswald's model $\left(\tau=K D^{n}\right)$ [9] applied to the rheograms of each suspension. $\tau$ is the shearing stress, $D$ the rate of shear and $K$ represents the viscosity of the preparation. The optimised suspension formulation requires a maximised dispersible sediment volume, an absence of non-redispersible caking and an appropriate viscosity for a good delivery.

The experimental domains are given in table I. The levels of the factors were selected according to the literature and from our experience [3]. The matrix and the responses for both drugs are reported in table II.

\section{Results and discussion}

\section{Sulfadiazine}

The quadratic models describing the responses are:

$$
\begin{aligned}
Y_{1}= & 7.43-5.01 X_{1}-5.54 X_{2}+29.78 X_{3}+4.70 X_{1} X_{1}- \\
& 10.5 X_{1} X_{2}-10.37 X_{1} X_{3}+3.81 X_{2} X_{2}-10.99 X_{2} X_{3} \\
& +20.74 X_{3} X_{3}
\end{aligned}
$$

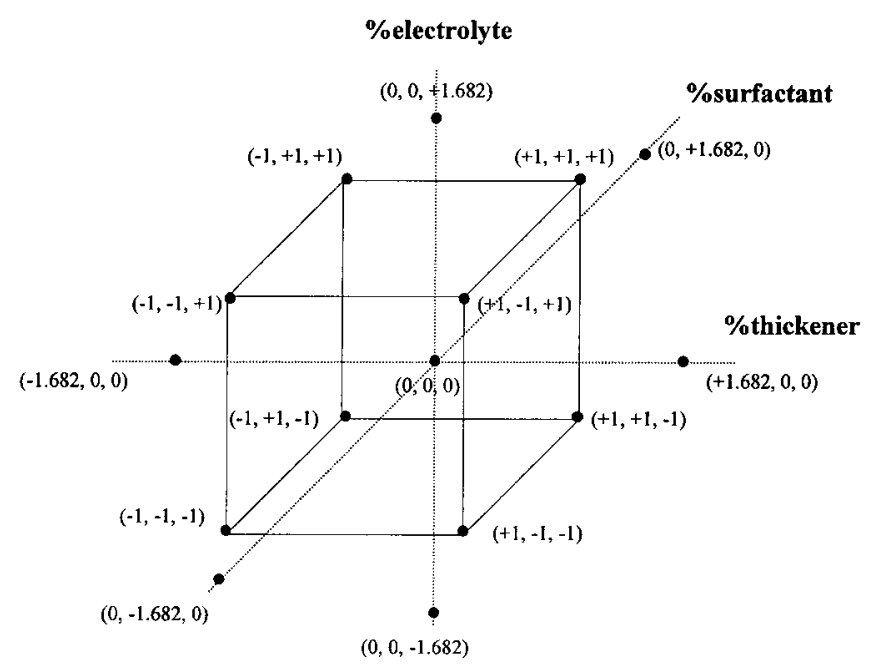

Fig. 1. Representation of a central composite design. A $2^{3}$ full factorial and a star design. 
$Y_{2}=10.71+1.105 X_{1}+1.52 X_{2}-7.12 X_{3}+0.515 X_{1} X_{1}+$ $0.875 X_{1} X_{2}+0.375 X_{1} X_{3}-0.37 X_{2} X_{2}-0.125 X_{2} X_{3}$ $-0.90 X_{3} X_{3}$.

An analysis of variance tested the statistical significance of each effect. In the case of both responses, one main effect, the effect of the factor "thickener", is significantly different from zero at the $95 \%$ confidence level. No interaction has a significant effect. The R-squared statistic shows that the model as fitted justifies $93.86 \%$ of the variability of the caking level and $88.32 \%$ in the case of sediment volume. We consider this last value as acceptable.

The graphical illustration of the results permits a good evaluation of what happens (Fig. 2). As the factor $X_{3}$ is the most significant, and since the variations of electrolyte and surfactant as a function of this factor give response surfaces with similar shapes, we decided to represent the response surfaces for sediment volume and caking level as a function of electrolyte and thickener. Figure 2 indicates that the maximum dispersed sediment volume is obtained for the highest thickener concentration and the lower electrolyte concentration. Figure 3 shows that the concentration of

Table I. Experimental domains and coding of the variables.

\begin{tabular}{lccccc}
\hline Variables & \multicolumn{5}{c}{ Levels } \\
& -1.682 & -1 & 0 & +1 & +1.682 \\
\hline$X_{1} \%$ electrolyte & 0 & 0.18 & 0.45 & 0.72 & 0.90 \\
$X_{2} \%$ surfactant & 0 & 0.41 & 1 & 1.60 & 2 \\
$X_{3} \%$ thickener & 0 & 0.304 & 0.750 & 1.196 & 1.50 \\
\hline Responses & $Y_{1}$ & \multicolumn{2}{l}{ sediment volume (\%) } \\
& $Y_{2}$ & caking level (ml) \\
& $Y_{1}^{\prime}$ & sediment volume (\%) \\
& $Y_{2}^{\prime}$ & viscosity (mPa.s) & \\
\end{tabular}

Table II. Matrix and responses for sulfadiazine and aluminium hydroxyde.

\begin{tabular}{|c|c|c|c|c|c|c|c|}
\hline \multirow[t]{3}{*}{ Run } & \multirow{2}{*}{\multicolumn{3}{|c|}{$\begin{array}{c}\text { Independent } \\
\text { Variables }\end{array}$}} & \multicolumn{4}{|c|}{ Responses } \\
\hline & & & & \multicolumn{2}{|c|}{ Sulfadiazine } & \multicolumn{2}{|c|}{ Al. Hydroxyde } \\
\hline & $X_{1}$ & $X_{2}$ & $X_{3}$ & $Y_{1}$ & $Y_{2}$ & $Y_{1}^{\prime}$ & $Y_{2}^{\prime}$ \\
\hline 1 & -1 & -1 & -1 & 10.11 & 17 & 23.3 & 0.036 \\
\hline 2 & 1 & -1 & -1 & 10.79 & 16 & 26.7 & 0.026 \\
\hline 3 & -1 & 1 & -1 & 13.21 & 18 & 27.9 & 0.034 \\
\hline 4 & 1 & 1 & -1 & 13.37 & 20 & 29.2 & 0.036 \\
\hline 5 & -1 & -1 & 1 & 96.47 & 0 & 100 & 3.62 \\
\hline 6 & 1 & -1 & 1 & 97.14 & 0 & 97.7 & 3.42 \\
\hline 7 & -1 & 1 & 1 & 94.14 & 0 & 100 & 3.59 \\
\hline 8 & 1 & 1 & 1 & 14.33 & 4 & 96.5 & 3.67 \\
\hline 9 & -1.682 & 0 & 0 & 6.47 & 10 & 88.2 & 0.96 \\
\hline 10 & +1.682 & 0 & 0 & 14.11 & 16 & 93 & 0.88 \\
\hline 11 & 0 & -1.682 & 0 & 7.55 & 7 & 97.6 & 0.874 \\
\hline 12 & 0 & +1.682 & 0 & 8.0 & 14 & 94.1 & 0.913 \\
\hline 13 & 0 & 0 & -1.682 & 11.3 & 18 & 15.1 & 0.007 \\
\hline 14 & 0 & 0 & +1.682 & 100.0 & 0 & 100 & 8.606 \\
\hline 15 & 0 & 0 & 0 & 5.74 & 12 & 92.9 & 0.943 \\
\hline 16 & 0 & 0 & 0 & 7.47 & 12 & 92.9 & 0.901 \\
\hline 17 & 0 & 0 & 0 & 8.52 & 10 & 95.9 & 1.086 \\
\hline 18 & 0 & 0 & 0 & 9.49 & 10 & 97.6 & 2.18 \\
\hline 19 & 0 & 0 & 0 & 7.86 & 10 & 96.4 & 2.05 \\
\hline 20 & 0 & 0 & 0 & 9.09 & 10 & 96.4 & 1.95 \\
\hline
\end{tabular}

electrolyte has no influence on the caking level, but that a high concentration of thickener limits consequentially the caking level. By superimposing the contourplots of both responses we can delimit the optimal zone to formulate the sulfadiazine suspensions (Fig. 4). The caking level should be

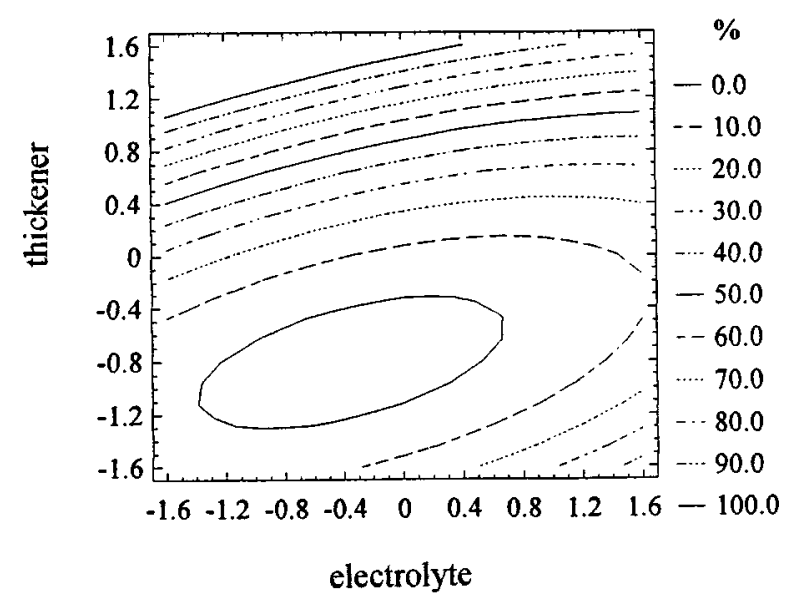

Fig. 2. Contours of estimated response surface for sediment volume of sulfadiazine suspensions.

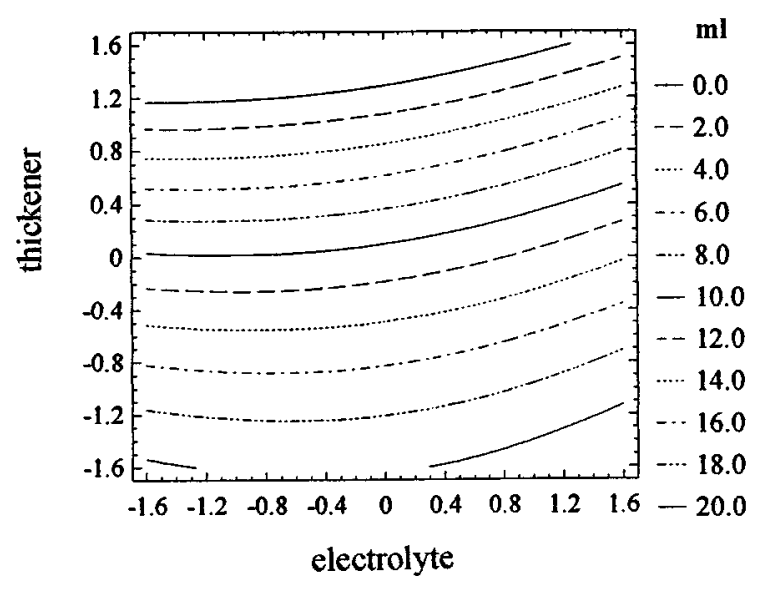

Fig. 3. Contours of estimated response surface for caking level of sulfadiazine suspensions.

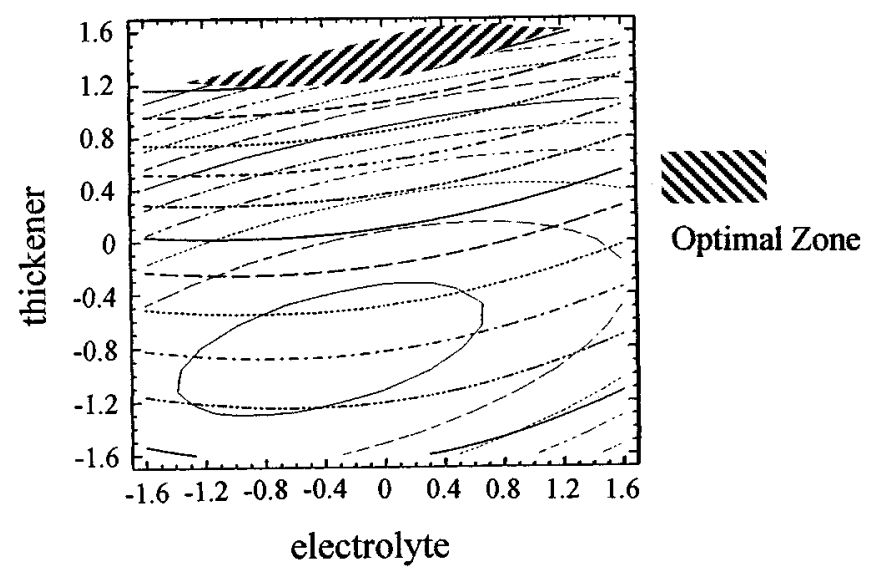

Fig. 4. Superimposed response contours of sediment volume and caking level for sulfadiazine suspensions. 


\section{Original articles}

equal to zero and the volume of dispersed sediment is considered acceptable between 90 and 100\%. These conditions should lead to homogeneous, easy to disperse and stable suspensions. An experiment were performed in the optimal zone corresponding to the proportions of electrolyte $0.45 \%$, surfactant $1 \%$ and thickener $1.35 \%$ and the results are conformable to the predicted values.

\section{Aluminium hydroxide}

The quadratic models describing the responses are:

$$
\begin{aligned}
Y_{1}^{\prime}= & 95.92+0.510 X_{1}+0.001 X_{2}+31.47 X_{3}-5.44 X_{1} X_{1} \\
& -0.41 X_{1} X_{2}-1.31 X_{1} X_{3}-3.58 X_{2} X_{2}-1.03 X_{2} X_{3} \\
& -17.12 X_{3} X_{3} \\
Y_{2}^{\prime}= & 1.52-0.018 X_{1}+0.02 X_{2}+2.09 X_{3}-0.27 X_{1} X_{1}+ \\
& 0.0365 X_{1} X_{2}-0.014 X_{1} X_{3}-0.277 X_{2} X_{2}+0.026 X_{2} \\
& X_{3}+0.92 X_{3} X_{3} .
\end{aligned}
$$

As for Sulfadiazine, the analysis of variance give the effect of the factor "thickener" significantly different from zero at the $95 \%$ confidence level, and also, no interaction has a significant effect. The R-squared statistics indicate that the model as fitted explains $92.65 \%$ of the variability of the sediment volume and $94.9 \%$ in the case of the viscosity. The graphical representations show the same sort of results for these responses. Figure 5 and figure 6 indicate that the concentration of electrolyte has no influence on the caking level (the surfactant gives same result), but that a high concentration of thickener gives the highest sediment volume and of course maximises the viscosity. By superimposing the contourplots of both responses we can delimit the optimal zone to formulate the Aluminium hydroxide suspensions (Fig. 7). We consider that the volume of dispersed sediment is good from 90 to $100 \%$ and the adequate deliverance will be achieved with a value of apparent viscosity between 1.5 and 3.5 Pa.s. This zone has been verified with an experimental point (electrolyte $0.45 \%$, surfactant $1 \%$, thickener $0.9 \%$ ) which lead to optimal properties.

These results are perfectly related with the properties of methylcellulose in suspensions. This sort of components can protect each particle in suspension and is able to limit the sedimentation. Their role of thickeners explains also the decrease of the sedimentation phenomenon [10]. The electrolyte and the surfactant have in both cases no significant effect, their effects are certainly masked by the preponderant influence of the methylcellulose.

\section{Conclusion}

This work has allowed to optimise the formulation of two drugs in suspension. The strategy chosen, a central composite design was suitable to point on the main significant effect and to determine the optimal conditions of formulation. We can see that the optimised areas were quite small and were situated near the limits of the domain. It would be interesting to develop the research in that region of the domain to make our formulation more robust.

\section{References}

1. Attwood, D.; Florence, A. T. Surfactant Systems, Chapman and Hall, London, 1983.

2. Swarbrick, J.; Boylan, J. C. Encyclopaedia of pharmaceutical tecnology, Marcel Dekker, New York, 1996.

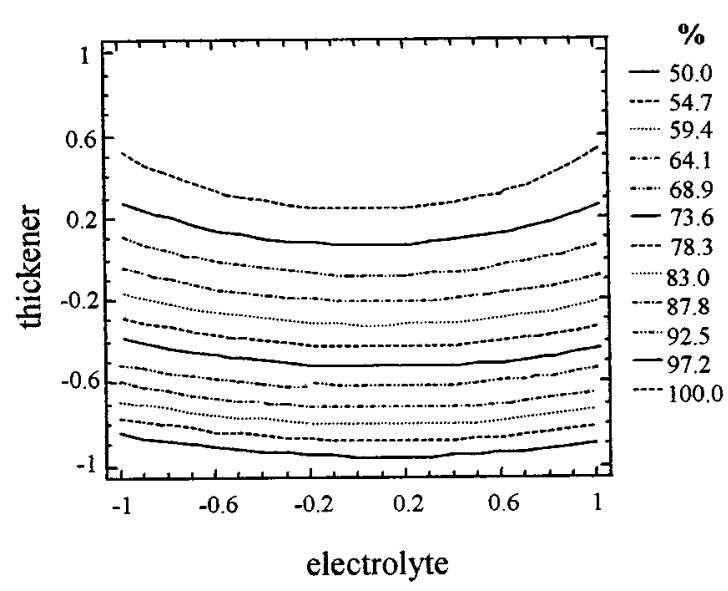

Fig. 5. Contours of estimated response surface for sediment volume of aluminium hydroxide suspensions.

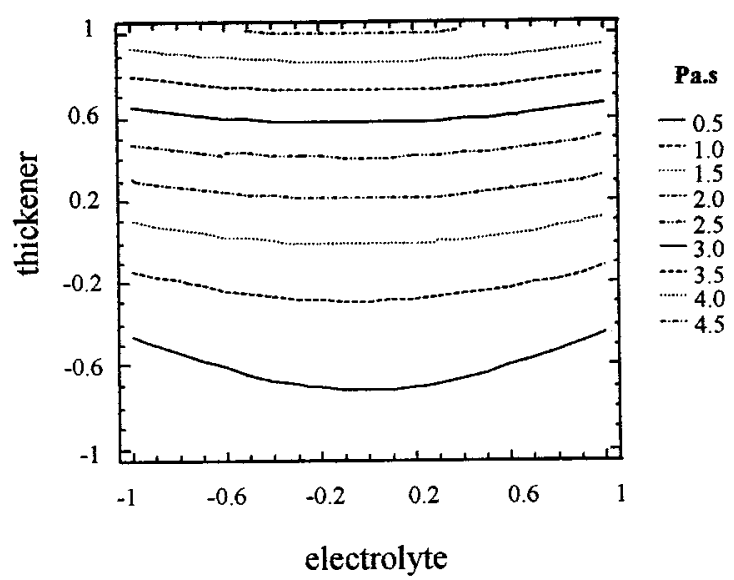

Fig. 6. Contours of estimated response surface for viscosity of aluminium hydroxide suspensions.

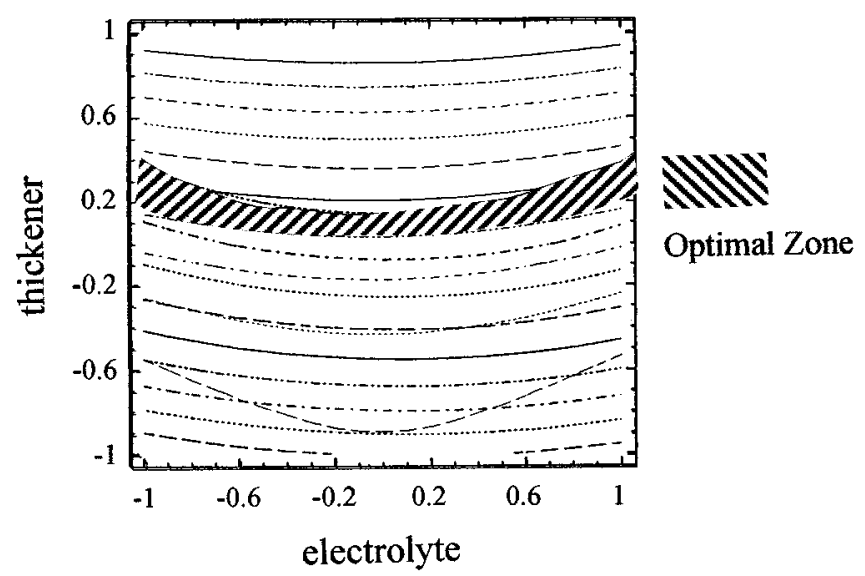

Fig. 7. Superimposed response contours of sediment volume and viscosity for aluminium hydroxyde suspensions.

3. Eyraud, M.; Plan d'expériences assisté par ordinateur pour la mise au point de formes pharmaceutiques. S.T.P. Pharma Pratique 1992, 2(5) 345-351.

4. Dictionnaire Vidal 1997, Éditions du Vidal, Paris, 1997.

5. Box, G. E.; Hunter, W. G.; Hunter, J. S. Response surface methods. Statistics for experimenters, Wiley \& Sons, New York, 1978. 
6. Benoist, D.; Tourbier, Y.; Germain-Tourbier, S. Plans d'expériences : construction et analyse, Technique \& Documentation, Lavoisier, 1994.

7. Cochran, G.; Cox, G. Experimental Designs, 2nd edition, Wiley \& Sons, New York, 1992.

8. STATGRAPHIC Plus, Manugistics, Inc. Maryland, U.S.A. 1995.
9. Couarraze G.; Grossiord, J.L. Initiation à la rhéologie, Technique \& Documentation - Lavoisier, Paris, 1991.

10. Nash, R. A. Pharmaceutical suspensions, in: Pharmaceutical dosage forms: Dispersed systems. Dekker, M., New York, 1988. 